\title{
Cytoskeletal staining in rectal cancer: problems
}

\author{
Viroj Wiwanitkit
}

Accepted: 24 June 2010 / Published online: 8 July 2010

(C) Springer-Verlag 2010

\section{Dear Editor:}

I read the recent publication "Cytokeratin staining for complete remission in rectal cancer after chemoradiation" by Gonullu et al. (Int J Colorectal Dis. 2010; 25: 805-809) with great interest. Gonullu et al. concluded that "cytokeratin IHC offers a means of improving staging accuracy and thus provides useful information for prognosis and treatment decisions for patients with rectal cancer who had a clinical CR after CRT." The problems of the techniques "falsenegative and/or false-positive results (Painter et al. Toxicol Patho. 2010; 38: 131-141)" should be mentioned. In a recent study on immostaining of sentinel lymph node mapping in colorectal cancer, false negative seems to be very clinically important (Sommariva et al. Eur J Surg Oncol.
2010;36:130-134). A mucinous histology seem to interfere with the reliability of staging (Sommariva et al. Eur J Surg Oncol. 2010;36:130-134). Nevertheless, the reported sensitivity and specificity of the cytokeratin staining in diagnosis of malignancy are about $80 \%$ and $75 \%$, which cannot be considered good (Golijianin et al. J Urol. 2000; 164: 1922-1925). Many factors including tissue type, fixation time, quality control on specimen collection and frozen sectioning, and antibody specificity are mentioned for their impact on the result of IHC (Painter et al. Toxicol Pathol. 2010; 38: 131-141). How to solve the basic diagnostic problems and control of quality of the whole IHC process is required before implementation of the staining technique for real usage

\footnotetext{
V. Wiwanitkit $(\bowtie)$

Wiwanitkit House, Bangkhae,

Bangkok, Thailand 10160

e-mail: wviroj@yahoo.com
} 